\title{
Advanced Manufacturing for Biomaterials and Biological Materials, Part I
}

\author{
DAVID RESTREPO, ${ }^{1,5}$ STEVEN E. NALEWAY, ${ }^{2,6}$ VINOY THOMAS, ${ }^{3,7}$ and \\ HANNES C. SCHNIEPP (10) \\ 1.-Department of Mechanical Engineering, The University of Texas at San Antonio, San Antonio, \\ TX 78249, USA. 2.-Department of Mechanical Engineering, University of Utah, Salt Lake City, \\ UT 84112, USA. 3.-Department of Materials Science and Engineering, University of Alabama at \\ Birmingham, Birmingham, AL 35294, USA. 4.-Department of Applied Science, William \& Mary, \\ PO Box 8795, Williamsburg, VA 23187, USA. 5.—e-mail: david.restrepo@utsa.edu. 6.—e-mail: \\ steven.naleway@mech.utah.edu. 7.—e-mail: vthomas@uab.edu. 8.—e-mail: schniepp@wm.edu
}

Manufacturing and processing are becoming increasingly important for biomaterials, bioinspired materials, and biological materials. This includes additive manufacturing techniques such as threedimensional (3D) printing, advanced manufacturing techniques such as freeze casting, and advanced materials processing methods based on microwaves and light. These techniques allow for increasing degrees of complexity, which is particularly helpful to mimic structures observed in natural materials. However, since biomaterials and biological materials require the use of particular materials classes and often have additional requirements, such as biocompatibility, significant research is required to implement these new manufacturing techniques. Many of these aspects are represented in this special topic on "Advanced Manufacturing for Biomaterials and Biological Materials". The first part of this special topic focuses on metallic materials, or materials containing metallic components.

The first two studies make use of metallic nanoparticles for new biomaterials. The first article, entitled "Effect of Al Addition on Structural, Magnetic, and Antimicrobial Properties of Ag Nanoparticles for Biomedical Applications", by El-Bassuony describes the synthesis of silver (Ag) and silver aluminate $\left(\mathrm{AgAlO}_{2}\right)$ nanoparticles (NPs) by a facile and low-cost flash method. The saturation magnetization and magnetic susceptibility of the $\mathrm{AgAlO}_{2}$ NPs were significantly higher than those of the Ag NPs. The coercivity of the $\mathrm{AgAlO}_{2} \mathrm{NPs}$ was several

Hannes C. Schniepp is the JOM advisor for the Biomaterials Committee, part of the TMS Functional Materials Division and Structural Materials Division, and guest editor for the topic Advanced Manufacturing for Biomaterials and Biological Materials Part I in this issue. David Restrepo, Steven E. Naleway, and Vinoy Thomas are Guest Editors for the Biomaterials Committee. times lower than that of the Ag NPs, which enables the application of such Ag NPs in magnetic targeting of tumors and separators, whereas the application of such $\mathrm{AgAlO}_{2} \mathrm{NPs}$ enables magnetic field sensors. Antimicrobial tests showed that the Ag and $\mathrm{AgAlO}_{2} \mathrm{NPs}$ exhibited significant activity against bacterial microorganisms; the Ag NPs also exhibited significant activity against fungal microorganisms. Therefore, $\mathrm{Ag}$ and $\mathrm{AgAlO}_{2}$ nanoparticles, obtained using this facile and low-cost method, are highly suitable for applications in antibacterial drugs, and the Ag NPs could also be applied as antifungal drugs.

The second manuscript, entitled "Study on Fe$x$ GO Composites Prepared by Selective Laser Melting: Microstructure, Hardness, Biodegradation and Cytocompatibility", addresses the problem that the degradation rate of pure iron is too slow for clinical applications. Therefore, in this work, powders of pure $\mathrm{Fe}$ and graphene oxide (GO) were used to prepare Fe- $x \mathrm{GO}$ composites $(x=0.4$ wt. $\%, 0.8$ wt. $\%$, 1.2 wt.\%, and 1.6 wt.\%) via selective laser melting (SLM), aiming to obtain a higher degradation rate. The degradation rate of the SLMed Fe- $x$ GO composites was faster than that of SLMed Fe, due to the incorporation of GO into the Fe. The GO content had a significant effect on the microstructure, hardness, and degradation rate. The SLMed Fe$0.8 \mathrm{GO}$ composite presented the finest, relatively uniform grains, the maximum degradation rate, density, and hardness, and good cytocompatibility. The mechanisms were also clarified.

The next manuscript, entitled "Effects of Stress Relieving Heat Treatment on the Impact Toughness of Direct Metal Laser Sintering (DMLS) Produced Ti6Al4V (ELI) Parts", by Amos Muiruri et al. investigated the effect of stress-relieving heat treatments 
on the Charpy impact toughness of DMLS metals when tested at temperatures that are physiologically relevant for the aircraft industry. The results demonstrate that stress-relieving heat treatment at $650^{\circ} \mathrm{C}$ both increased the impact toughness and lowered the temperature of the ductile-to-brittle transition when compared with as-built DMLS Ti6Al4V metals. The resulting impact toughness was found to be below the requirements for the aircraft industry, but the results demonstrate that the microstructural refinement induced by stressrelieving heat treatments in DMLS Ti6Al4V metals can improve their impact resistance.

Plasma sintering was the subject of "Optimization of Spark Plasma Sintering Parameters Using the Taguchi Method for Developing Mg-Based Composites", an original research paper by Hussain et al., who explored this technique for processing of both metals and ceramic composite biomaterials. The authors fabricated magnesium-based metal matrix composites incorporated with 2.5 wt.\% $\mathrm{TiB}_{2}$ using spark plasma sintering (SPS) for the first time. The Taguchi design approach was used to analyze the significant influences of sintering parameters, such as the temperature, pressure, and time, on the physical and mechanical properties of the Mg-based composites. The authors concluded that, in the case of microhardness, the sintering temperature was the most significant factor among all the studied process parameters.

In their manuscript "A Materials Perspective on the Design of Damage-Resilient Bone Implants through Additive/Advanced Manufacturing," Le Ferrand and Athanasiou discuss current limitations in the manufacturing and development of bone implants. The leading causes of bone implant failure include brittle fracture of the implant itself, or the fracture of the bone underneath the implant due to the mismatch between the mechanical properties of the bone and implant. The degraded mechanical performance of current commercial bone implants stems from a failure to reproduce the multiscale organization of natural bone, where the local hierarchical microstructure enables multiscale toughening mechanisms. For instance, the structure and stress distribution in native hips exhibit a mesh of tension and compression lines that develop perpendicular to each other, ultimately leading to zero stress at the center of the bone, which prevents catastrophic failure. In contrast, most current implants are homogeneous microscopically and do not exhibit these stiffening and toughening mechanisms. This review highlights that advanced and additive manufacturing methods provide strategies in this direction, bridging the gaps between natural tissues and engineering implants. The lack of unified testing approaches is a major showstopper for bench-to-bed translation, and the authors conclude that new experimental frameworks for mechanical characterization of inhomogeneous biological and biomimicking materials need to be developed.

In "Microwave Processing of Biomaterials for Orthopedic Implants: Challenges and Possibilities," Sharma and Gupta review microwave material processing, whose application in the biomaterials field remains largely unexplored, as a time- and energy-efficient technique for biomaterials processing, especially for orthopedic materials. In this review article, developments on various biomaterials used in arthroplasty are reported extensively, along with their processing using microwave energy. Furthermore, challenges involved in their processing with a brief outline of various possibilities are highlighted for this biomaterials manufacturing method.

The manuscript "A Review on Additive Manufacturing of Shape-Memory Materials for Biomedical Applications" by Nasim Sabahi et al. discusses the importance of shape-memory materials (SMMs) in biomedical engineering. SMMs are stimulus-sensitive materials that can be deformed and fixed in a temporary stable shape, then recover their stressfree original form upon exposure to an appropriate stimulus, such as temperature, electric field, $\mathrm{pH}$ change, and near-infrared light. Shape-memory alloys (SMAs) and shape-memory polymers (SMP) are the two most widely used classes of SMMs. NiTi alloys are the most widely used SMAs in the biomedical field due to their excellent functional properties, including large recovery strains and biocompatibility, high corrosion resistance, low stiffness, and excellent compatibility with magnetic resonance. NiTi alloys have been used in many biomedical fields, including orthopedics, endodontics, orthodontics, neurology, vascular, and medical instruments. SMPs have been widely used in tissue engineering, drug delivery, endovascular surgery, orthodontics, and orthopedics. This review highlights that the use of SMMs as feedstock materials for additive manufacturing (AM) has driven a new layer in 3D printing, being known as four-dimensional (4D) printing, where fabricated parts can be actuated and transformed after fabrication. This development has brought about tremendous opportunities in biomedical applications by allowing mass customization of devices and implants to be patient specific.

To read or download any of the papers from this topic, follow the URL http://link.springer.com/journ al/11837/72/3/page/ 1 to the Table of Contents page for the March 2020 issue (vol. 72, no. 39).

\section{ACKNOWLEDGEMENTS}

D.R. acknowledges funding by the San Antonio Area Foundation, and UTSA VPREDKE. S.E.N. acknowledges funding by the National Science Foundation under Grant No. CMMI-1660979. V.T. 
acknowledges funding by the National Science Foundation through NSF EPSCoR RIITrack-1 Cooperative Agreement OIA-1655280. H.C.S. acknowledges funding by the National Science Foundation under Grant Nos. DMR-1352542, DMREF-1534428, and DMR-1905902.
Publisher's Note Springer Nature remains neutral with regard to jurisdictional claims in published maps and institutional affiliations. 